\title{
Prediction of T-cell epitopes of hepatitis C virus genotype $5 a$
}

\author{
Maemu P Gededzha, M Jeffrey Mphahlele and Selokela G Selabe*
}

\begin{abstract}
Background: Hepatitis C virus (HCV) is a public health problem with almost 185 million people estimated to be infected worldwide and is one of the leading causes of hepatocellular carcinoma. Currently, there is no vaccine for $\mathrm{HCV}$ infection and the current treatment does not clear the infection in all patients. Because of the high diversity of $\mathrm{HCV}$, protective vaccines will have to overcome significant viral antigenic diversities. The objective of this study was to predict T-cell epitopes from HCV genotype 5a sequences.
\end{abstract}

Methods: HCV near full-length protein sequences were analyzed to predict T-cell epitopes that bind human leukocyte antigen (HLA) class I and HLA class II in HCV genotype 5a using Propred I and Propred, respectively. The Antigenicity score of all the predicted epitopes were analysed using VaxiJen v2.0. All antigenic predicted epitopes were analysed for conservation using the IEDB database in comparison with 406, 221, 98, 33, 45,45 randomly selected sequences from each of the HCV genotypes 1a, 1b, 2, 3, 4 and 6 respectively, downloaded from the GenBank. For epitope prediction binding to common HLA alleles found in South Africa, the IEDB epitope analysis tool was used.

Results: A total of 24 and 77 antigenic epitopes that bind HLA class I and HLA class II respectively were predicted. The highest number of HLA class I binding epitopes were predicted within the NS3 (63\%), followed by NS5B (21\%). For the HLA class II, the highest number of epitopes were predicted in the NS3 (30\%) followed by the NS4B (23\%) proteins. For conservation analysis, 8 and 31 predicted epitopes were conserved in different genotypes for HLA class I and HLA class II alleles respectively. Several epitopes bind with high affinity for both HLA class I alleles and HLA class II common in South Africa.

Conclusion: The predicted conserved T-cell epitopes analysed in this study will contribute towards the future design of HCV vaccine candidates which will avoid variation in genotypes, which in turn will be capable of inducing broad HCV specific immune responses.

Keywords: Hepatitis C virus, Genotype 5a, In-silico, T-cell epitopes, Vaccines

\section{Introduction}

Hepatitis C virus (HCV) is estimated to infect 185 million people worldwide [1]. Chronic HCV infection leads to progressive liver disease, being one of the major causes of hepatocellular carcinoma and one of the most common indications for liver transplantation [2]. The World Health Organization (WHO) strongly recommends combination therapy with pegylated interferon and ribavirin for chronically infected patients who qualify for treatment

\footnotetext{
* Correspondence: selokela.selabe@ul.ac.za

HIV and Hepatitis Research Unit, Department of Virology, University of
Limpopo, Medunsa Campus/National Health Laboratory Service, Pretoria,

HIV and Hepatitis Research Unit, Department of Virology, University of
Limpopo, Medunsa Campus/National Health Laboratory Service, Pretoria, South Africa
}

[1]. Recently, two NS3 protease inhibitors (boceprevir and telaprevir) have been approved by the US Food and Drug Administration, and the WHO conditionally (until more evidence has accumulated) recommends that these drugs should be given in combination with pegylated interferon and ribavirin for the treatment of chronic $\mathrm{HCV}$ genotype 1 infections. Also, the WHO strongly recommends that sofosbuvir be given in combination with ribavirin alone in patients who cannot tolerate interferon and are chronically infected with genotypes 1, 2, 3 and 4 [1]. However, these therapies are still not affordable in most developing countries.

\section{Biomed Central}

(c) 2014 Gededzha et al.; licensee BioMed Central Ltd. This is an Open Access article distributed under the terms of the Creative Commons Attribution License (http://creativecommons.org/licenses/by/4.0), which permits unrestricted use, distribution, and reproduction in any medium, provided the original work is properly credited. The Creative Commons Public Domain Dedication waiver (http://creativecommons.org/publicdomain/zero/1.0/) applies to the data made available in this article, unless otherwise stated. 
As a result, the development of an effective $\mathrm{HCV}$ vaccine is undoubtedly the best solution for the ultimate control of HCV infections, and is a public health priority.

Prophylactic vaccines against viral infections are generally aimed at inducing a humoral (B-cell) immune response, while therapeutic vaccines preferably activate both humoral and cellular (T-cell) immune responses [3]. A successful $\mathrm{HCV}$ vaccine will need to stimulate both arms of the adaptive immune response, since while both cellular and humoral immune responses occur in a naturally infected host, the current consensus is that a strong cellular response is vital for viral clearance and protection [4].

The development of an effective HCV vaccine requires an understanding of the host's adaptive immune response to natural infection. As with other viral infections, viral antigens are presented to CD4+ and CD8+ T-cells via human leukocyte antigen (HLA) class II and class I molecules, respectively [5]. Different HLA class alleles have been found to be associated with $\mathrm{HCV}$ infection. For example, HLA-A*11, HLA-Cw*04 and HLA-B*53 have been associated with $\mathrm{HCV}$ persistence [6,7], while HLA-B*27, HLA-A*11:01, HLA-B*57, HLA-Cw*01:02 and HLA-A*03 have been associated with spontaneous HCV clearance $[8,9]$. Also, HLA-DRB1*11 and HLADQB1*03:01 have been associated with decreased disease severity of $\mathrm{HCV}$ infection globally, suggesting they may present $\mathrm{HCV}$-derived epitopes more efficiently to CD4+ T-cells than others and thus capable of viral clearance [10]. During acute HCV infection, development and persistence of strong specific responses by CD8+ and CD4+ T-cells [11,12] and neutralizing antibodies [13] are associated with viral clearance, with HCV-specific CD8+ and CD4+ T-cells usually being transient or absent in patients who develop persistent infections. The rate of chronic liver disease progression has been shown to be determined by the magnitude of HCV-specific CD4+ T-cell responses, since these cells are essential for both the cellular and humoral responses [14]. CD8+ T-cells are essential for long-term protection against chronic $\mathrm{HCV}$ [15], while CD4+ T-cells play a role in viral clearance [16].

$\mathrm{HCV}$ infection evades the host's immune system by generating immune escape variants through alteration of the virus HLA-restricted epitopes to avoid being recognized by T-cells and neutralizing antibodies [14]. Thus effective HCV vaccines will need to target protective epitopes that display minimal cross-genotype amino acid variability as they will provide broad potency [17]. Peptides corresponding to protective epitopes are desirable vaccine candidates because they are easy to construct and produce, and they do not contain infectious materials [18]. The first step in the process of epitope-based vaccine design and development is the in-silico prediction of peptide binding affinities to HLA proteins [19].

Genotype 5 accounts for over $50 \%$ of HCV infections in South Africa [20], and is becoming more prevalent in Europe and North America [21]. It is the most conserved of HCV genotypes, being classified into only one subtype (5a) [22-24]. The growing prevalence of $\mathrm{HCV}$ genotype $5 \mathrm{a}$ in different parts of the world necessitates its molecular characterization in order to improve the formulations of vaccine candidates that are in development. Thus the aim of this study was to assess immunological determinants by predicting conserved epitopes in near-full length $\mathrm{HCV}$ genotype $5 \mathrm{a}$ sequences using a suite of online programmes to help in the designing of new vaccine candidates.

\section{Results}

\section{Prediction of T-cell epitopes}

For HLA class I, a total of 24 antigenic epitopes were predicted in the consensus near full-length of genotype 5a (Table 1). Epitope NS3 ${ }^{1325-1333}$ covered 30 of the 47 HLA class I alleles that were analysed assuring high binding affinity to different alleles. For conservation analysis with other genotypes, 8 of 24 epitopes were $100 \%$ conserved for specific genotypes. Epitopes $\mathrm{NS}^{1332-1340}$ and NS5B $\mathrm{B}^{2557-2565}$ were highly conserved in all genotypes analysed, and epitope $\mathrm{E} 2^{684-692}$ was conserved in all genotypes except for genotype 2, while 5 other epitopes were conserved in either 2 or 3 genotypes analysed. In addition epitope NS4B ${ }^{1832-1840}$ was conserved in genotype $1 \mathrm{a}, 1 \mathrm{~b}, 2$ and 4 epitope variants at anchor residues position 2 and 9 while E2 ${ }^{677-685}$, NS3 ${ }^{1325-1333}$ and NS3 ${ }^{1357-1365}$ were conserved in at least 3 genotypes epitope variants each (Table 1). For HLA class II, 77 epitopes were predicted (Table 2). Epitope NS4B ${ }^{1879-1887}$ and NS4B ${ }^{1880-1888}$ covered 51 of 51 HLA class II alleles analysed. For conservation analysis with other genotypes, 31 of 71 epitopes were conserved for specific genotypes. Some epitopes were highly conserved in all genotypes $\left(\mathrm{E}^{507-515}, \mathrm{E} 2^{509-517}, \mathrm{NS}^{1253-1261}\right.$, $\mathrm{NS}^{1254-1262}, \mathrm{NS}^{1327-1335}, \mathrm{NS}^{1392-1400}, \mathrm{NS}^{13 B^{1916-1924}}$ and $\mathrm{NS}_{4} \mathrm{~B}^{1919-1927}$ ), while other epitopes were conserved in at least 1 to 4 of the genotypes analysed. Epitope NS4B ${ }^{1886-1894}$ was conserved at anchor residues position 1, 4, 6 and 9 in all 6 genotypes epitope variants while $\mathrm{E} 2^{692-700}, \mathrm{NS}^{964-972}$, NS3 ${ }^{1418-1426}$ and NS4 ${ }^{1561-1569}$ were conserved in at least 5 genotypes epitope variants each (Table 2). Epitopes NS3 ${ }^{1585-1593}$, NS5A $^{2285-2293}$ and NS5B ${ }^{2889-2897}$ were predicted to cover both HLA class I and HLA class II alleles. The highest number of HLA class I binding epitopes were predicted within the NS3 (63\%), followed by NS5B (21\%), and for the HLA class II, the highest number of 
Table 1 HLA class I predicted epitopes of HCV genotype 5a and their antigenicity prediction score, number of allele and conservation (in percentages) in different genotypes

\begin{tabular}{|c|c|c|c|c|c|c|c|c|c|}
\hline Position & Epitope sequence & No of allele & Antigenecity score & Genotype 1a & Genotype 1b & Genotype 2 & Genotype 3 & Genotype 4 & Genotype 6 \\
\hline \multicolumn{10}{|l|}{ E2 } \\
\hline 651 & RCDLEDRDR & 9 & 2.7428 & & & & & & \\
\hline 677 & CSFTPTPAL & 27 & 0.8772 & CSFT几PAL (94) & CSFT几PAL (92) & & CSFTPMPAL (73) & & \\
\hline 684 & ALSTGLIHL $^{\#}[25-27]$ & 29 & 0.9005 & ALSTGLIHL (70) & ALSTGLIHL (99) & ALSTGLIHL (97) & ALSTGLIHL (97) & ALSTGLIHL (84) & ALSTGLIHL (91) \\
\hline \multicolumn{10}{|l|}{ NS2 } \\
\hline 861 & VPPLQVRGG & 6 & 1.4312 & VPPLMVRGG (85) & & & & & \\
\hline \multicolumn{10}{|l|}{ NS3 } \\
\hline 1032 & YAQQTRGVL & 26 & 0.7638 & YAQQTRGLL (99) & & & & & \\
\hline 1033 & AQQTRGVLG & 5 & 0.7751 & AQQTRGLLG (99) & & & & & \\
\hline 1128 & ADLYLVTRH & 5 & 1.0277 & SDLYLVTRH (99) & SDLYLVTRH (99) & VDLYLVTRN (92) & & & \\
\hline 1325 & TILGIGTVL & 30 & 0.7555 & SILGIGTVL (93) & & TILGIGTVL (88) & SILGIGTVL (94) & TILGIGTVL (82) & \\
\hline 1332 & VLDQAETAG & 5 & 0.5004 & VLDQAETAG (96) & VLDQAETAG (98) & VLDQAETAG (96) & VLDQAETAG (100) & VLDQAETAG (93) & VLDQAETAG (71) \\
\hline 1357 & TPHPNIEEV & 24 & 0.8423 & VPHPNIEEV (89) & VPHPNIEEV (80) & & VPHSNIEEV (94) & & \\
\hline 1359 & HPNIEEVAL $^{\#}$ [28-31] & 24 & 1.1654 & HPNIEEVAL (88) & HPNIEEVAL (76) & & HSNIEEVAL (94) & & \\
\hline 1370 & EGEIPFYGR & 9 & 1.0560 & TGEIPFYGK (97) & & & & & \\
\hline 1374 & PFYGRAIPL & 7 & 0.9479 & PFYGKAIPL (99) & PFYGKAIPI (85) & PFYGKAIPL (82) & & & PFYGKAIPL (73) \\
\hline 1642 & TKYIMACMS & 6 & 0.7008 & PFYGKAIPL (99) & TKYIMACMS (70) & & TKYIMACMS (70) & TKYIMACMS (93) & TKYIMTCMS (87) \\
\hline \multicolumn{10}{|l|}{ NS4B } \\
\hline 1712 & SASLPYMDE & 5 & 0.5488 & & & & & & \\
\hline 1819 & QIAPPTAAT & 5 & 0.5293 & QLAAPGAAT (99) & QLAPPSAAS (94) & QIAPPAGAT (91) & & QIATPTAST (89) & \\
\hline 1832 & SGMAGAAVG & 5 & 0.8750 & AGLAGAA/G (81) & AG/AGAAVG (93) & SGLVGAAVG (98) & SGLAGAA/G (76) & & \\
\hline 1848 & LIDILAGYG & 5 & 0.8431 & LVDILAGYG (94) & LVDILAGYG (95) & & LLDILAGYG (79) & LVDILAGYG (84) & \\
\hline 1854 & GYGAGVAGA & 9 & 0.5562 & GYGAGVAGA (99) & GYGAGVAGA (99) & & GYGAGVSGA (94) & GYGAGVAGA (91) & GYGAGVSGA (84) \\
\hline \multicolumn{10}{|l|}{ NS5B } \\
\hline 2522 & GYGAKEVRS & 7 & 1.3540 & GYGAKDVRC (95) & GYGAKDVRN (84) & GFGAKEVRS (93) & GYSAKDVRS (79) & & \\
\hline 2557 & TIMAKNEVF $^{\#}[32]$ & 12 & 0.6303 & TIMAKNEVF (99) & TIMAKNEVF (80) & TIMAKNEVF (83) & TIMAKNEVF (100) & TIMAKNEVF (84) & TIMAKNEVF (93) \\
\hline 2568 & EPSKGGKKP & 6 & 1.4218 & QPEKGGRKP (93) & & & & & \\
\hline 2720 & LASCRAAKL & 28 & 0.5264 & & & & & & \\
\hline 2886 & LHGLSAFSL & 11 & 0.6732 & LHGLSAFSL (99) & LHGLSAFSL (98) & & & LHGLSAFT(82) & LHGMAAFSL (98) \\
\hline
\end{tabular}

"-indicates that the epitope has been experimentally proven to be a true positive.

Bold- indicates percentage of epitope that is $100 \%$ conserved in more than $70 \%$ of the sequences analysed in each genotype. Italic- indicates amino acid(s) variation in epitope in comparison to the predicted epitope. 
Table 2 HLA class II predicted epitopes of HCV genotype 5a and their antigenicity prediction score, number of allele and conservation (in percentages) in different genotypes

\begin{tabular}{|c|c|c|c|c|c|c|c|c|c|}
\hline Position & Epitope sequence & $\begin{array}{l}\text { No of } \\
\text { allele }\end{array}$ & $\begin{array}{l}\text { Antigenecity } \\
\text { score }\end{array}$ & Genotype 1a & Genotype 1b & Genotype 2 & Genotype 3 & Genotype 4 & Genotype 6 \\
\hline \multicolumn{10}{|l|}{ E1 } \\
\hline 320 & WDMMMNWSP & 10 & 1.3610 & WDMMMNWSP (99) & WDMMMNWSP (97) & & WDMMMNWSP (97) & WDMMMNWSP (93) & WDMMMNWSP (93) \\
\hline \multicolumn{10}{|l|}{ E2 } \\
\hline 507 & YCFTPSPVV & 6 & 1.2112 & YCFTPSPVV (93) & YCFTPSPVV (98) & YCFTPSPVV (88) & YCFTPSPVV (100) & YCFTPSPVV (91) & YCFTPSPVV (91) \\
\hline 509 & FTPSPVVVG & 17 & 1.3553 & FTPSPVVVG (94) & FTPSPVVVG (98) & FTPSPVVVG (88) & FTPSPVVVG (100) & FTPSPVVVG (89) & FTPSPVVVG (73) \\
\hline 665 & LLHTTTQWA & 5 & 0.6616 & LLLSTTQWQ (100) & & & LLHSTTELA (73) & LLLSTTQWQ (73) & \\
\hline 666 & LHTTTQWAI & 25 & 0.5863 & LLTTTQWQI (100) & & & LHSTTELAI (73) & & \\
\hline 692 & LHQNIVDTQ & 6 & 0.8564 & LHQNIVDVQ (100) & LHQNIVDVQ (73) & LHQNIVDVQ (95) & LHQNIVDVQ (100) & LHQNIVDVQ (87) & \\
\hline 739 & LLVCQAEAA & 22 & 0.5506 & LLISQAEAA (100) & LLIAQAEAA (72) & & LMISQAEAA (73) & & \\
\hline \multicolumn{10}{|l|}{ P7 } \\
\hline 803 & LPHRALALD & 7 & 0.6966 & LPQRAYALD (100) & LPPRAYAMD (86) & & & & \\
\hline \multicolumn{10}{|l|}{ NS2 } \\
\hline 860 & WVPPLQVRG & 7 & 1.1998 & WVPPLNRG (100) & & & WVPPLLARG (82) & & \\
\hline 861 & VPPLQVRGG & 25 & 1.4312 & VPPLMRGG (100) & & & & & \\
\hline 864 & LQVRGGRDA & 20 & 0.6368 & LNRRGGRDA (100) & LMRGGRDA (86) & & & & \\
\hline 880 & FHPALGFEI & 19 & 1.4374 & VHPALVFDI (99) & & & & & \\
\hline 892 & LLGILGPLY & 14 & 0.5940 & LLAИGPLW (100) & & & LIAИGPLY (82) & & \\
\hline 895 & ILGPLYLLQ & 5 & 0.8264 & ИGPLWUQ (99) & & & ИLGPLYL/Q (88) & & \\
\hline 938 & LLHLGRLTG & 25 & 0.5554 & & & & & & \\
\hline 964 & LRDLAVATE & 9 & 1.0497 & LRDLAVAVE (100) & LRDLAVAVE (93) & LRDLAVAVE (85) & LKDLAVATE (73) & & LRDLAVAVE (93) \\
\hline 997 & LAGLPVSAR & 11 & 0.9120 & INGLPVSAR (100) & & & LCGLPVSAR (100) & & \\
\hline 1025 & LLAPITAYA & 22 & 0.5253 & LLAPITAYA (99) & & & LLAPITAYA (79) & LLAPITAYA (89) & \\
\hline \multicolumn{10}{|l|}{ NS3 } \\
\hline 1047 & LTGRDKNEA & 22 & 1.5633 & LTGRDKNQV (100) & LTGRDKNQV (93) & & LTGRDKNW (90) & & \\
\hline 1131 & YLVTRHADV $^{\#}[33-35]$ & 15 & 0.7569 & YLVTRHADV (99) & YLVTRHADV (99) & YLVTRNADV (97) & & & \\
\hline 1152 & LLSPRPISY & 5 & 2.2999 & LLSPRPISY (99) & LLSPRPVSY (73) & LLSPRPLST (89) & & & \\
\hline 1153 & LSPRPISYL & 5 & 1.3109 & LSPRPISYL (99) & LSPRPVSYL (73) & LSPRPLST (89) & & & \\
\hline 1253 & VLNPSVAAT & 14 & 1.2489 & VLNPSVAAT (100) & VLNPSVAAT (100) & VLNPSVAAT (97) & VLNPSVAAT (100) & VLNPSVAAT (96) & VLNPSVAAT (89) \\
\hline 1254 & LNPSVAATL & 6 & 0.9169 & LNPSVAATL (100) & LNPSVAATL (100) & LNPSVAATL (98) & LNPSVAATL (100) & LNPSVAATL (95) & LNPSVAATL (89) \\
\hline 1258 & VAATLGFGA & 11 & 1.2617 & VAATLGFGA (90) & VAATLGFGA (85) & VAATLGFGA(98) & & & \\
\hline 1262 & LGFGAYMSK" [36-38] & 14 & 0.8784 & LGFGAYMSK (87) & LGFGAYMSK (82) & LGFGAYMSK(78) & & & \\
\hline
\end{tabular}


Table 2 HLA class II predicted epitopes of HCV genotype 5a and their antigenicity prediction score, number of allele and conservation (in percentages) in different genotypes (Continued)

\begin{tabular}{|c|c|c|c|c|c|c|c|c|c|}
\hline 1264 & FGAYMSKAY & 14 & 0.6561 & FGAYMSKAH (100) & FGAYMSKAH (82) & & & & \\
\hline 1327 & LGIGTVLDQ & 42 & 0.5607 & LGIGTVLDQ (97) & LGIGTVLDQ (88) & LGIGTVLDQ (98) & LGIGTVLDQ (100) & LGIGTVLDQ (93) & LGIGTVLDQ (96) \\
\hline 1373 & IPFYGRAIP & 16 & 0.5679 & IPFYGKAIP (100) & IPFYGKAIP (93) & IPFYGKAIP (81) & & & \\
\hline 1375 & FYGRAIPLA & 22 & 1.0280 & FYGKAIPLE (100) & FYGKAIPIE (81) & & & & FYGKAIPLE (71) \\
\hline 1392 & IFCHSKKKC & 22 & 1.5437 & IFCHSKKKC (93) & IFCHSKKKC (94) & IFCHSKKKC (95) & IFCHSKKKC (88) & IFCHSKKKC (80) & IFCHSKKKC (93) \\
\hline 1415 & VAYYRGLDV & 21 & 0.8250 & VAYYRGLDV (98) & VAYYRGLDV (96) & VAYYRGLDV (92) & VAYYRGLDV (79) & VAYYRGLDV (91) & VAFYRGVDV (89) \\
\hline 1418 & YRGLDVAVI & 51 & 1.0522 & YRGLDVSVI (100) & YRGLDVSVI (97) & YRGLDVSVI (84) & YRGLDVSVI (85) & YRGLDVSVI (91) & YRGVDVSVI (67) \\
\hline 1482 & VSRSQRRGR & 12 & 2.0649 & VSRTQRRGR (100) & VSRSQRRGR (93) & VSRSQRRGR (96) & VSRSQRRGR (97) & VSRSQRRGR (98) & VSRSQRRGR (73) \\
\hline 1561 & VFTGLTNID & 9 & 1.1689 & VFTGLTHID (100) & VFTGLTHID (96) & VFTGLTHID (98) & VFTGLTHID (97) & & VFTGLTHID (89) \\
\hline 1583 & FPYLVAYQA & 24 & 0.6584 & & FPYLVAYQA (85) & & & & FAYLVAYQA (78) \\
\hline 1585 & YLVAYQATV $^{\#}[25,39]$ & 17 & 0.5020 & YLVAYQATV (99) & YLVAYQATV (85) & YLTAYQATV (70) & & YLVAYQATV (87) & YLVAYQATV (91) \\
\hline 1586 & LVAYQATVC & 28 & 0.6052 & LVAYQATVC (99) & LVAYQATVC (85) & & & LVAYQATVC (82) & LVAYQATVC (91) \\
\hline 1631 & VQNEITLTH & 35 & 1.1979 & VQNEVTLTH (100) & & & & VQNEVTLTH (80) & \\
\hline 1641 & ITKYIMACM & 8 & 0.6964 & VTKYIMTCM (100) & & VTKYIATCM (94) & & ITKYIMACM (75) & ITKYIMTCM (87) \\
\hline 1645 & IMACMSADL & 22 & 0.8406 & IMTCMSADL (100) & IMACMSADL (82) & IATCMQADL (99) & IMACMSADL (70) & IMACMSADL (93) & IMTCMSADL (87) \\
\hline \multicolumn{10}{|l|}{ NS4B } \\
\hline 1733 & LGLIGTAGQ & 43 & 1.2450 & & & & & & \\
\hline 1765 & MWNFVSGIQ & 18 & 1.2300 & MWNFISGIQ (100) & MWNFISGIQ (95) & MWNFISGIQ (96) & MWNFVSGIQ (100) & MWNFISGIQ (87) & \\
\hline 1766 & WNFVSGIQY & 22 & 0.6246 & WNFISGIQY (100) & WNFISGIQY (95) & WNFISGIQY (96) & WNFVSGIQY (100) & WNFISGIQY (87) & WNFVSGIQY (91) \\
\hline 1791 & MSFTAAVTS & 8 & 0.7414 & MAFTAAVTS (100) & MAFTASTS (88) & MAFSAALTS (93) & MAFTASVTS (92) & MSFTAAVTS (93) & \\
\hline 1812 & LGGWVASQI & 16 & 0.608 & LGGWVAQL (100) & LGGWAAQL (95) & & LGGWATHL (88) & LGGWVASQI (96) & \\
\hline 1849 & IDILAGYGA & 9 & 0.7525 & VDILAGYGA (100) & VDILAGYGA (97) & & LDILAGYGA (79) & VDILAGYGA (84) & \\
\hline 1863 & LVAFKIMCG & 47 & 1.0160 & LVAFKIMSG (100) & & LVAFKIMSG (95) & LVAFKIMGG (100) & WTFKIMSG (89) & LVAFKIMSG (84) \\
\hline 1879 & LVNLLPSIL & 51 & 0.7592 & LVNLLPAIL (100) & LVNLLPAIL (90) & & MVNLLPAIL (92) & LVNLLPAIL (73) & \\
\hline 1880 & VNLLPSILC & 51 & 0.9874 & VNLLPAILS (100) & VNLLPAILS (92) & & VNLLPAILS (94) & VNLLPAILS (89) & VNLLPALLS (82) \\
\hline 1883 & LPSILCPGA & 6 & 0.7104 & LPAILSPGA (100) & LPAILSPGA (99) & LPAILSPGA (74) & LPAILSPGA (100) & LPAILSPGA (89) & LPALLSPGA (84) \\
\hline 1886 & ILCPGALVV & 9 & 0.9365 & ILSPGALW (100) & ILSPGALW (99) & ILSPGALW (98) & ILSPGALW (100) & ILSPGALW (91) & LLSPGALW (89) \\
\hline 1892 & LVVGVICAA & 30 & 0.7097 & LWGVVCAA (100) & LWGVVCAA (98) & LVVGVICAA (97) & LVVGVICAA (94) & LWGVVCAA (89) & LWGVVCAA (93) \\
\hline 1893 & VVGVICAAV & 50 & 0.9530 & WGVVCAA/ (100) & WGVVCAAI (96) & WGVICAAI (98) & WGVICAAI (85) & WGVVCAAI (91) & WGVVCAAI (76) \\
\hline 1896 & VICAAVLRR & 35 & 0.9887 & VVCAAILRR (100) & VVCAAILRR (95) & VICAA/LRR (97) & VICAA/LRR (85) & VVCAA/LRR (91) & VVCAAILRR (76) \\
\hline 1897 & ICAAVLRRH & 12 & 1.2316 & VCAAILRRH (100) & VCAAILRRH (97) & ICAAILRRH (97) & ICAA/LRRH (85) & VCAA/LRRH (89) & \\
\hline 1916 & MNRLIAFAS & 48 & 0.5291 & MNRLIAFAS (100) & MNRLIAFAS (99) & MNRLIAFAS (99) & MNRLIAFAS (100) & MNRLIAFAS (93) & MNRLIAFAS (96) \\
\hline
\end{tabular}


Table 2 HLA class II predicted epitopes of HCV genotype 5a and their antigenicity prediction score, number of allele and conservation (in percentages) in different genotypes (Continued)

\begin{tabular}{|c|c|c|c|c|c|c|c|c|c|}
\hline 1919 & LIAFASRGN & 24 & 2.0235 & LIAFASRGN (100) & LIAFASRGN (99) & LIAFASRGN (98) & LIAFASRGN (100) & LIAFASRGN (93) & LIAFASRGN (96) \\
\hline 1922 & FASRGNHVS & 18 & 1.2938 & FASRGNHVS (100) & FASRGNHVS (98) & FASRGNHVA (97) & FASRGNHVS (94) & FASRGNHVS (82) & FASRGNHVS (93) \\
\hline \multicolumn{10}{|c|}{ NS5A } \\
\hline 1994 & WLQAKLLPQ & 33 & 1.1603 & WLKAKLMPQ (100) & WLQSKLLPR (84) & & & & \\
\hline 2101 & YITGVTQDN & 6 & 1.1358 & YVTGMTTDN (100) & & & & & \\
\hline 2105 & VTQDNLKCP & 6 & 1.0178 & MTTDNLKCP (100) & & & & & \\
\hline 2237 & MGGNITRVE & 20 & 1.2656 & MGGNITRVE (92) & MGGNITRVE (96) & & MGSNITRVE (91) & & \\
\hline 2285 & LPVWARPGY & 9 & 0.6143 & LPMARPDY (100) & & & & & LPMARPDY (78) \\
\hline \multicolumn{10}{|c|}{ NS5B } \\
\hline 2422 & MSYSWTGAL & 9 & 0.5749 & MSYSWTGAL (76) & MSYTWTGAL (94) & MSYSWTGAL (97) & MSYSWTGAL (85) & MSYSWTGAL (91) & \\
\hline 2451 & LRHHNLVYS & 40 & 1.2321 & LRHHNLVYS (89) & LRHHNMVYA (86) & & LRHHNLVYS (97) & & \\
\hline 2523 & YGAKEVRSL & 24 & 1.1739 & YGAKDVRCH (100) & YGAKDVRNL (85) & FGAKEVRSL (92) & YSAKDVRSL (76) & & \\
\hline 2559 & MAKNEVFAV & 18 & 0.8466 & MAKNEVFCV (100) & MAKNEVFCV (81) & MAKNEVFCV (65) & MAKNEVFCV (85) & & MAKNEVFCV (96) \\
\hline 2564 & VFAVEPSKG & 10 & 0.7925 & VFCVQPEKG (100) & VFCVQPEKG (89) & & & & \\
\hline 2637 & FSYDTRCFD & 11 & 15808 & FSYDTRCFD (99) & & FSYDTRCFD (95) & FSYDTRCFD (100) & FSYDTRCFD (93) & FSYDTRCFD (87) \\
\hline 2660 & YQSCDLQPE & 11 & 1.3802 & YQCCDLDPQ (100) & YQCCDLAPE (94) & & & & \\
\hline 2696 & YRRCRASGV & 41 & 1.2642 & YRRCRASGV (99) & YRRCRASGV (99) & YRRCRASGV (98) & YRRCRASGV (97) & & \\
\hline 2778 & YDLELVTSC & 6 & 1.0923 & YDLEL/TSC (100) & YDLEL/TSC (96) & YDLEL/TSC (98) & YDLEL/TSC (91) & & YDLEL/TSC (96) \\
\hline 2850 & FSVLQSQEQ & 21 & 0.8451 & & FS/LLAQEQ (84) & & & FS/LQSQEA (78) & \\
\hline 2871 & VYSVTPLDL & 29 & 2.0113 & CYSIEPLDL (100) & CYSIEPLDL (75) & & TYSVTPLDL (91) & TYSTTPLDL (87) & \\
\hline 2883 & IQRLHGLSA & 50 & 0.4322 & IQRLHGLSA (98) & IQRLHGLSA (72) & IQYLAGLST (93) & IERLHGLSA (79) & IQRLHGLSA (75) & IQRLHGMAA (96) \\
\hline 2889 & LSAFSLHSY $^{\#}[40]$ & 6 & 0.6007 & LSAFSLHSY (99) & LSAFSLHSY (97) & & & LSAFてLHGY (80) & MAAFSLHGY (89) \\
\hline
\end{tabular}

\#-indicates that the epitope has been experimentally proven to be a true positive.

Bold- indicates percentage of epitope that is $100 \%$ conserved in more than $70 \%$ of the sequences analysed in each genotype. Italic- indicates amino acid(s) variation in epitope in comparison to the predicted epitope. 
epitopes were predicted in the NS3 (30\%) followed by the NS4B (23\%) proteins (Table 3).

\section{Epitope binding affinity to common South African HLA class I and HLA class II alleles}

Genotype 5 epitopes and their genotypic variants were analysed for their binding affinity to HLA class I and HLA class II alleles most common in South Africa, where genotype $5 \mathrm{a}$ is predominating. For HLA class I, 11 of the most common South African HLA-A alleles (HLA-A*01:01, HLA-A*02:01, HLA-A*30:01 and HLA$A * 30: 02)$, HLA-B (HLA-B*0702, HLA-B*08:01 and HLA-B*3501), and HLA-C (HLA-C*04:01, HLA-C*06:01, HLA-C*07:01 and HLA-C*07:02) were analysed. The limitation of Propred 1 is that it does not cover most of the main HLA class I alleles: HLA-A (HLA-A*01:01, HLA$A * 30: 01$ and HLA-A*30:02), HLA-B (HLA-B*08:01) and HLA-C (HLA-C*04:01, HLA-C*06:01, HLA-C*07:01 and HLA-C*07:02) that are observed in South Africa. As a results the IEDB epitope analysis tool was used to predict epitopes of all the 11 most common HLA-A, HLA-B, HLA-C covering HLA class I alleles found in South Africa with the ANN prediction server. Thirteen antigenic epitopes with high binding affinity score of $<50 \mathrm{IC}_{50} \mathrm{nM}$ were predicted. Most epitopes bind with high affinity to single HLA class I alleles with exception of epitope NS5B ${ }^{2889-2297}$ LSAFSLHSY that bind with high affinity to 2 HLA-A alleles (HLA-A*01:01 and HLA-A*30:02). Four of the epitopes binding to HLA-B*35:01 followed by HLA-A*02:01 with 3 epitopes binding to it, however, none of the epitopes bind to HLA-C*04:01, HLA-C*06:01 and HLA-C*07:02 alleles. The NS3 ${ }^{1359-1367}$ shows a level of promiscuity to HLA-A and HLA-B alleles. NS3 ${ }^{1359-1367}$ HPNIEEVAL bind with intermediate affinity to HLA-B*07:02, while its genotype 2, 3 and 4 variant HSNIEEVAL bind with poor affinity and genotype 6 variant HPNITETAL bind with high affinity. For HLA-B*35:01, the NS3 ${ }^{1359-1367}$ HPNIEEVAL and genotype 6 variant HPNITETAL bind with high affinity while genotype 2, 3 and 4 variant HSNIEEVAL bind with poor affinity. Three $\left(\mathrm{E} 2^{684-692}, \mathrm{NS} 3^{1032-1040}\right.$ and NS3 ${ }^{1359-1367}$ ) of the thirteen epitopes were predicted by both Propred 1 and IEDB analysis tool (Table 4).

For HLA class II alleles, 4 most common HLA-DRB alleles (HLA-DRB1*03:01, HLA-DRB1*04:01, HLA-DRB1*11:01 and HLA-DRB1*15:01) were analysed. Nineteen antigenic epitopes with high binding affinity score of $<50 \mathrm{IC}_{50} \mathrm{nM}$ were predicted. The HLA-DRB1*11:01 has the highest number of binding epitopes (10) followed by HLADRB1*04:01 and HLA-DRB1*15:01 (7 each). Epitopes NS4B ${ }^{1919-1927}$ LIAFASRGN was predicted to be the most promiscuous epitope binding to HLA-DRB1*04:01, HLADRB1*11:01 and HLA-DRB1*15:01 with high affinity and HLA-DRB1"03:01 with intermediate affinity. This epitope is conserved in all genotypes. Epitopes $\mathrm{E} 2^{507-515}$, $\mathrm{NS}^{\mathrm{B} 1774-1782}$, NS4B ${ }^{1919-1927}$, and NS4B ${ }^{1920-1928}$ were highly conserved in all genotypes (Table 5).

\section{Validation of epitopes}

Seven of the predicted epitopes were previously confirmed experimentally by other studies as true positives in comparison with the epitopes analysed in the IEDB resource database. Majority of the epitopes predicted in this study have not been previously tested experimentally. The 'true epitopes' are highlighted by $\left({ }^{\#}\right)$ in Tables 1,2 and 4 .

\section{Discussion}

Several studies that have published HCV epitopes focused mainly on genotype 1 [41,42], but most of these studies do not take into account the diversity in other genotypes that are common in developing countries like most African countries. In the present study, predicted antigenic epitopes of HCV genotype 5a proteins from South Africa were analysed followed by conservation with randomly selected genotypes 1-6 references from GenBank. Several studies have confirmed the importance of using immunoinformatics as good predictors for selecting HLA ligands, Tcell epitopes and immunogenicity [43]. As a result, several immunoinformatics methods have been developed to assist in the identification of HLA binding peptides [44,45].

For this analysis, near full-length sequences covering all $\mathrm{HCV}$ proteins with the exclusion of the 3 ' end of the NS5B were included to maximize number of epitopes predicted. The use of the whole viral genome for developing epitope vaccines has a potential control over the immune response and eliminating the side effects [43], and it also increases the chance of detecting a virus at any developmental stage [46]. It has been shown that multiple epitopes from different parts of the HCV genome are important to produce a vaccine that can elicit strong humoral immune responses and multiple specific cellular

Table 3 Distribution of genotype 5a HLA class I and II predicted epitopes in each of the HCV gene

\begin{tabular}{cccccccccccc}
\hline HLA class & No of predicted epitopes & \multicolumn{8}{c}{ Distribution of the predicted epitopes in each of the HCV gene a $^{\mathbf{c}}$} \\
\cline { 2 - 13 } & & $\mathbf{C}$ & E1 & E2 & P7 & NS2 & NS3 $^{\mathbf{b}}$ & NS4A & NS4B & NS5A & NS5B \\
\hline I & 24 & $0(0)$ & $0(0)$ & $3(12)$ & $0(0)$ & $1(4)$ & $\mathbf{1 5 ( 6 3 )}$ & $0(0)$ & $0(0)$ & $0(0)$ & $5(21)$ \\
$\|$ & 77 & $0(0)$ & $1(1)$ & $6(8)$ & $1(1)$ & $10(13)$ & $\mathbf{2 3 ( 3 0 )}$ & $0(0)$ & $18(23)$ & $5(7)$ & $13(7)$ \\
\hline
\end{tabular}

${ }^{a}$ Number of epitopes and their percentage in brackets.

${ }^{\mathrm{b}} \mathrm{HCV}$ gene with highest number of binding epitopes (in bold). 
Table 4 Binding affinity scores of predicted epitopes and their variants to common HLA I allele types prevalent in South Africa

\begin{tabular}{|c|c|c|c|c|c|c|c|c|c|c|c|c|c|}
\hline \multirow[t]{3}{*}{ Gene } & \multirow[t]{3}{*}{ Epitope sequence ${ }^{a}$} & \multirow[t]{3}{*}{ Genotype of epitope } & \multicolumn{11}{|c|}{ HLA I allele types ${ }^{b}$} \\
\hline & & & \multicolumn{4}{|c|}{ HLA-A } & \multicolumn{3}{|c|}{ HLA-B } & \multicolumn{4}{|c|}{ HLA-C } \\
\hline & & & $\begin{array}{c}\text { HLA- } \\
A^{*} 01: 01\end{array}$ & $\begin{array}{c}\text { HLA- } \\
A^{*} 02: 01\end{array}$ & $\begin{array}{c}\text { HLA- } \\
A^{*} 30: 01\end{array}$ & $\begin{array}{c}\text { HLA- } \\
A^{*} 30: 02\end{array}$ & $\begin{array}{c}\text { HLA- } \\
B^{*} 07: 02\end{array}$ & $\begin{array}{c}\text { HLA- } \\
B^{*} 08: 01\end{array}$ & $\begin{array}{c}\text { HLA- } \\
B^{*} 35: 01\end{array}$ & $\begin{array}{c}\text { HLA- } \\
\text { C*04:01 }\end{array}$ & $\begin{array}{c}\text { HLA- } \\
C^{*} 06: 02\end{array}$ & $\begin{array}{c}\text { HLA- } \\
\text { C*07:01 }\end{array}$ & $\begin{array}{c}\text { HLA- } \\
\text { C*07:02 }\end{array}$ \\
\hline
\end{tabular}

\begin{tabular}{lcc}
\hline E2 & & \\
684 & ALSTGLIHL $^{\#}[25-27]$ & 1a, 1b, 3, 4,5a, 6 \\
& ALSTGLLHL & 2
\end{tabular}

NS2

$1025 \quad$ LLAPITAYA 3, 4, 5a

LLAPITAYT 2

NS3

YAQQTRGLL 1a, 2

YSQQTRGLL $1 \mathrm{~b}$

YAQQTRGLV 6

$\begin{array}{ccc}1242 & \text { AAYAAQGYK } & 1 \mathrm{a}, 1 \mathrm{~b}, 4,5 \mathrm{a} \\ 1264 & \text { AAYASQGYK } & 2 \\ & \text { FGAYMSKAY } & 5 \mathrm{a} \\ 1359 & \text { FGAYMSKAH } & 1 \mathrm{a}, 1 \mathrm{~b}, 2 \\ & \text { HPNIEEVAL }^{*}[28-31] & 1 \mathrm{a}, 1 \mathrm{~b}, 5 \mathrm{a} \\ & \text { HSNIEEVAL } & 2,3,4 \\ & \text { HPNITETAL } & 6 \\ & \text { LPSEGEIPF } & 5 \mathrm{a} \\ & \text { LGHEGEIPF } & 2 \\ & \text { LGSEGEIPF } & 3 \\ & \text { LPTTGEIPF } & 4,6\end{array}$

1585 YLVAYQATV $^{\#}[25,39] \quad 1 \mathrm{a}, 1 \mathrm{~b}, 4,5 \mathrm{a}, 6$ YLTAYQATV 2,3

$\begin{array}{ccc}\text { NS5A } & & \\ 1927 & \text { NHVSPTHYV } & 1 \mathrm{a}, 1 \mathrm{~b}, 3,4,5 \mathrm{a}, 6 \\ & \text { NHVAPTHYV } & 2 \\ 2285 & \text { LPWWARPGY } & 5 \mathrm{a} \\ & \text { LPNARPDY } & 1 \mathrm{a}, 3,4,6 \\ & \text { LPAWARPDY } & 2\end{array}$


Table 4 Binding affinity scores of predicted epitopes and their variants to common HLA I allele types prevalent in South Africa (Continued)

\begin{tabular}{|c|c|c|c|c|c|c|c|c|c|c|c|c|c|}
\hline \multicolumn{14}{|c|}{ NS5B } \\
\hline 2696 & YRRCRASGV & $1 a, 1 b, 2,3,5 a$ & + & + & + & + & + & ++ & + & + & ++ & +++ & ++ \\
\hline 2763 & MTRYSAPPG & $1 \mathrm{a}, 1 \mathrm{~b}, 2,3,4,5 \mathrm{a}, 6$ & + & + & +++ & + & + & + & + & + & + & + & + \\
\hline \multirow[t]{3}{*}{2889} & LSAFSLHSY" [40] & $1 a, 1 b, 5 a$ & +++ & + & + & +++ & + & + & ++ & + & + & + & + \\
\hline & LSAFてHSY & 3 & +++ & + & + & +++ & + & + & ++ & + & + & + & + \\
\hline & LSAFてLHGY & 4 & +++ & + & + & +++ & + & + & + & + & + & + & + \\
\hline
\end{tabular}

${ }^{\#}$-indicates that the epitope has been experimentally proven to be a true positive.

a-italics indicates amino acid(s) variation in epitope in comparison to the predicted epitope.

$\mathrm{b}_{+++}$indicates high binding affinity $\left(<50 \mathrm{I} \mathrm{I}_{50} \mathrm{~nm}\right)$, ++ indicates medium binding affinity $\left(>50 \mathrm{I} \mathrm{I}_{50} \mathrm{~nm},<500 \mathrm{I} \mathrm{I}_{50} \mathrm{~nm}\right)$, + indicates poor binding affinity $\left(>500 \mathrm{I} \mathrm{C}_{50} \mathrm{~nm}\right)$. 
Table 5 Binding affinity scores of predicted epitopes and their variants to common HLA class II allele types prevalent in South Africa

\begin{tabular}{|c|c|c|c|c|c|c|}
\hline \multirow[t]{2}{*}{ Position } & \multirow[t]{2}{*}{ Predicted epitopes } & \multirow[t]{2}{*}{ Genotype of epitope } & \multicolumn{4}{|c|}{ HLA II- DRB1 allele types ${ }^{a}$} \\
\hline & & & HLA-DRB $1 * 03: 01$ & HLA-DRB $1 * 04: 01$ & HLA-DRB $1 * 11: 01$ & HLA-DRB1*15:01 \\
\hline \multicolumn{7}{|l|}{ E2 } \\
\hline 507 & YCFTPSPW & $1 a, 1 b, 2,3,4,5 a, 6$ & + & +++ & + & +++ \\
\hline 695 & NIVDTQYLY & $5 a$ & + & +++ & + & +++ \\
\hline \multicolumn{7}{|l|}{ NS2 } \\
\hline 938 & LLHLGRLTG & $5 a$ & + & ++ & +++ & + \\
\hline 1025 & LLAPITAYA & $1 a, 3,4,5 a$ & + & ++ & +++ & ++ \\
\hline \multicolumn{7}{|l|}{ NS3 } \\
\hline 1129 & DLYLVTRHA & $1 a, 1 b, 5 a$ & + & + & +++ & + \\
\hline 1131 & YLVTRHADV & $1 a, 1 b, 5 a$ & + & + & +++ & + \\
\hline 1391 & LIFCHSKKK & $1 b, 2,3,4,5 a, 6$ & + & + & +++ & + \\
\hline 1417 & YYRGLDVAV & $5 a$ & + & +++ & ++ & + \\
\hline 1464 & FSLDPTFTI & $1 a, 1 b, 2,5 a$ & + & +++ & +++ & + \\
\hline 1535 & TTVRLRAYL & $3,5 a, 6$ & + & + & + & +++ \\
\hline 1562 & FTGLTNIDA & $5 a$ & + & +++ & ++ & + \\
\hline 1666 & WAALAAYC & $5 a$ & + & + & + & +++ \\
\hline \multicolumn{7}{|l|}{ NS4B } \\
\hline 1774 & YLAGLSTLP & $1 a, 1 b, 2,3,4,5 a, 6$ & + & +++ & + & + \\
\hline 1919 & LIAFASRGN & $1 a, 1 b, 2,3,4,5 a, 6$ & ++ & +++ & +++ & +++ \\
\hline 1920 & IAFASRGNH & $1 a, 1 b, 2,3,4,5 a, 6$ & + & + & +++ & + \\
\hline \multicolumn{7}{|l|}{ NS5A } \\
\hline 1994 & WLQAKLLPQ & $5 a$ & + & ++ & +++ & + \\
\hline 2099 & YHYITGVTQ & $5 a$ & + & ++ & +++ & ++ \\
\hline \multicolumn{7}{|l|}{ NS5B } \\
\hline 2450 & LLRHHNLVY & $1 a, 3,5 a$ & + & ++ & ++ & +++ \\
\hline 2579 & LIVYPDLGV & $2,3,4,5 a, 6$ & + & + & + & +++ \\
\hline
\end{tabular}

${ }_{+++}$indicates high binding affinity $\left(<50 \mathrm{IC} 5{ }_{50} \mathrm{~nm}\right),++$ indicates medium binding affinity $\left(>50 \mathrm{I} C_{50} \mathrm{~nm},<500 \mathrm{IC}{ }_{50} \mathrm{~nm}\right),+$ indicates poor binding affinity $\left(>500 \mathrm{I} C_{50} \mathrm{~nm}\right)$.

immune responses [47]. A polyepitope-based strategy with multiple components combining core, E1, and E2 proteins; and conserved T-cell epitopes in the NS proteins has been suggested to be a good vaccine candidate for HCV [48].

High number of epitopes was predicted for HLA class II as compared to class I. The findings of this study are consistent with a study by Shehzadi et al. that predicted epitopes in genotype 3 from Pakistan. The study showed that majority of predicted epitopes were found in the NS3 protein for both HLA class I and HLA class II alleles and most of the epitopes were conserved among different genotypes [49]. Although the NS3 region is one of the conserved regions in $\mathrm{HCV}$, variability in the nucleotide and amino acids has been reported by several studies in the same genotype and also in different genotypes [50,51]. A recent study that analysed 1568 NS3-protease sequences from genotypes 1-6 reported that the protease amino acids sequence was moderately conserved and majority of the amino acids clustered in small regions. Of the 181 amino acids analysed $47 \%$ showed $<1 \%$ variability among all $\mathrm{HCV}$ genotypes, and $17.1 \%$ amino acid positions showing $>25.1 \%$ variability [51]. The NS3 is considered to be a good cellular target candidate for a therapeutic vaccine [52] since majority of the HCV viral epitopes recognized by CD8+ and CD4+ T-cells are located in the NS3 region [53-56]. The NS3 specific CD4+ and $\mathrm{CD} 8+\mathrm{T}$-cell responses were reported in patient responders to interferon therapy [57] and in spontaneous clearance of HCV [58].

Most of the predicted epitopes in the study sequence were found to be conserved across different HCV genotypes with a higher number of epitopes conserved at the anchor residues. The anchor residues are important for epitope high binding affinity to HLA [59]. Conserved epitopes might influence the immunogenic potential 
since mutations within the epitopes can increase the chance of immune escape [60]. For a vaccine to be effective globally the selected epitopes must cover HLAs of different populations and it must also be conserved among different genotypes. The high mutation rates of viral epitopes and HLA polymorphisms are some of the challenges that are associated with the development of peptide vaccines [61]. Successful epitope vaccine design requires a broad knowledge of $\mathrm{HCV}$ genotype diversity. This will help in the proper selection of conserved HCV-specific T-cell epitopes that will help in avoiding $\mathrm{HCV}$ immune evasion [62]. This study attempted to ensure maximal coverage of HLA polymorphism and different genotypes by analyzing conserved epitopes considering different HLA alleles.

Majority of the epitopes predicted from HCV proteins isolated from South African genotype 5a were good binders against HLA alleles that are found worldwide. HLA is both polygenic and polymorphic, and the pool of HLA molecules differs for every individual. Different HLA alleles bind peptides with a particular sequence pattern [63]. For an HLA allele to be covered by a set of epitopes, at least one of the epitopes should be capable of inducing an immune response when bound to the corresponding HLA molecule [46]. The epitopes predicted in this study bind to many HLA alleles including the ones common in South Africa and can be used for designing good vaccine candidates that will eventually work in genetically diverse populations. In-vitro and in-silico studies have showed that HLA alleles preferentially bind to conserved regions of viral proteins in human viruses [64].

Very few epitopes were found to be experimentally true positive, however this can be due to the fact that most of the previous studies focused on genotype 1 . A limitation of the study was a lack of in-vivo and in-vitro studies to confirm the predicted immunogenic epitopes, which will be the focus of future studies. However in-silico studies still provide the basis for designing good vaccine candidates.

In conclusion, the results of this study demonstrated antigenic T-cell epitopes that are conserved among genotypes and good HLA binders derived from genotype 5 a sequences that can be good candidates for vaccine development. Predicted epitopes analysed in this study will contribute to the future design of an efficient vaccine with the use of conserved epitopes to avoid variation in genotypes and as such, it will be able to induce broad $\mathrm{HCV}$ specific immune responses. Conserved epitopes among different genotypes will be experimentally tested in the future to determine their involvement in immune response.

\section{Methods}

\section{Ethical statement}

The study was approved by the Medunsa Research and Ethics Committee of the Faculty of Health Sciences at the University of Limpopo as project no MREC/p/142/
2009:PG. The MREC is registered as an Independent Review Board with a reference no (IRB00005122).

\section{Prediction of T-cell epitopes}

Genotype 5a full-length sequences available in the GenBank and 6 of the near full length sequences generated from a previous study conducted by our group [24] were aligned and consensus sequences created using BioEdit [65] for the prediction of T-cell epitopes. For HLA class I, prediction for binding alleles was performed using ProPred I (http://www.imtech.res.in/raghava/propred1/) at a $4 \%$ default threshold by keeping the proteosome and immunoproteosome filters on at $5 \%$ threshold. ProPred 1 predicts antigenic epitopes for 47 HLA class I alleles [44]. For HLA class II, prediction was performed using ProPred (http://www.imtech.res.in/raghava/propred/) at a 3\% default threshold. ProPred predicts antigenic epitopes for 51 HLA class II alleles [45].

\section{Antigenicity of the epitopes}

The Antigenicity score of all the predicted epitopes were analysed using VaxiJen v2.0 online antigen prediction (www.ddg-pharmfac.net/vaxijen/). Epitopes having antigenic score $>0.5$ were selected as antigenic. Vaxijen server performed well with $87 \%$ accuracy at a threshold of 0.5 antigenic score for viruses. VaxiJen v2.0 allows antigen classification based on the physicochemical properties of proteins without recourse to sequence alignment.

\section{Epitope conservation analysis}

All predicted epitopes were analyzed for conservation using the IEDB database (http://tools.immuneepitope.org/ tools/conservancy/iedb_input) at a threshold of $100 \%$ conservation in comparison with 406, 221, 98, 33, 45, 45 randomly selected sequences from each of the HCV genotypes 1a, 1b, 2, 3, 4 and 6 respectively. The epitopes were considered conserved in another genotype if it shows $100 \%$ identity across the epitope in at least $70 \%$ of sequences in that genotype in the randomly selected sequences used in this study, downloaded from the public database. In addition, epitope variants that were conserved in at least $70 \%$ of the sequences were analysed for conservancy for anchor residues at positions 2 and 9 for HLA class I and positions 1, 4, 6 and 9 for HLA class II.

\section{Validation of predicted epitopes}

All the predicted epitopes were submitted to IEDB database (http://www.immuneepitope.org/) to confirm if they had been tested previously by other studies. The immuneepitope database contains experimentally confirmed data about antibody, T-cell epitopes, HLA binding, HLA restriction and HLA class. 


\section{Common South African HLA alleles}

For epitope prediction binding to common HLA alleles found in South Africa, the IEDB epitope analysis tool (http://tools.immuneepitope.org/tools/conservancy/iedb input) was used for HLA class I using the artificial neural network (ANN) algorithm [66] on the IEDB server, while for Class II ProPred (http://www.imtech.res.in/raghava/ propred/) at a 3\% default threshold was used. ProPred predicts antigenic epitopes for 51 HLA class II alleles [54]. The most common South African alleles were found in published literature [67].

\section{Abbreviations}

HCV: Hepatitis C virus; HLA: human leukocyte antigen.

\section{Competing interests}

The authors declare that they have no competing interests.

\section{Authors' contributions}

MPG designed the study, performed the immunoinformatics analysis and drafted the manuscript. SGS and MJM guided the project and critically reviewed the manuscript. All the authors have read and approved the final manuscript.

\section{Acknowledgements}

This work was supported by grants from Medical Research Council, National Research Foundation, National Health Laboratory Service Research Trust, and The Stella \& Paul Loewestein Charitable \& Educational Trust in South Africa.

Received: 17 March 2014 Accepted: 14 October 2014

Published: 8 November 2014

\section{References}

1. World health Organization [WHO]: Guidelines for the screening care and treatment of persons with hepatitis C infection. 2014, 1-122 [http://www. who.int/hiv/pub/hepatitis/hepatitis-c-guidelines/en/]

2. Pawlotsky JM: The nature of interferon-alpha resistance in hepatitis $C$ virus infection. Curr Opin Infect Dis 2003, 16:587-592.

3. Ip PP, Nijman HW, Wilschut J, Daemen T: Therapeutic vaccination against chronic hepatitis C virus infection. Antiviral Res 2012, 96:36-50.

4. Ashfaq UA, Javed T, Rehman S, Nawaz Z, Riazuddin S: An overview of HCV molecular biology, replication and immune responses. Virol J 2011, 8:161.

5. Singh R, Kaul R, Kaul A, Khan K: A comparative review of HLA associations with hepatitis B and C viral infections across global populations. World J Gastroenterol 2007, 13:1770-1787.

6. Thio CL, Nolt KR, Astemborski J, Vlahov D, Nelson KE, Thomas DL: Screening for hepatitis $C$ virus in human immunodeficiency virus-infected individuals. J Clin Microbiol 2000, 38(2):575-577.

7. Fanning $L$, Kenny-Walsh E, Shanahan F: Persistence of hepatitis C virus in a white population: associations with human leukocyte antigen class 1. Hum Immunol 2004, 65:745-751.

8. McKiernan SM, Hagan R, Curry M, McDonald GS, Kelly A, Nolan N, Walsh A Hegarty J, Lawlor E, Kelleher D: Distinct MHC class I and II alleles are associated with hepatitis $C$ viral clearance, originating from a single source. Hepatology 2004, 40:108-114.

9. Neumann-Haefelin C, McKiernan S, Ward S, Viazov S, Spangenberg HC, Killinger T, Baumert TF, Nazarova N, Sheridan I, Pybus O, von Weizsäcker F, Roggendorf M, Kelleher D, Klenerman P, Blum HE, Thimme R: Dominant influence of an HLA-B27 restricted CD8+ T cell response in mediating HCV clearance and evolution. Hepatology 2006, 43:563-572.

10. Hong $X$, Yu RB, Sun NX, Wang B, Xu YC, Wu GL: Human leukocyte antigen class II DQB1*0301, DRB1*1101 alleles and spontaneous clearance of hepatitis C virus infection: a meta-analysis. World J Gastroenterol 2005, 11:7302-7307.

11. Lechner F, Wong DK, Dunbar PR, Chapman R, Chung RT, Dohrenwend P, Robbins G, Phillips R, Klenerman P, Walker BD: Analysis of successful immune responses in persons infected with hepatitis $C$ virus. J Exp Med 2000, 191:1499-1512.
12. Spada E, Mele A, Berton A, Ruggeri L, Ferrigno L, Garbuglia AR, Perrone MP, Girelli G, Del Porto P, Piccolella E, Mondelli MU, Amoroso P, Cortese R, Nicosia A, Vitelli A, Folgori A: Multispecific T cell response and negative HCV RNA tests during acute HCV infection are early prognostic factors of spontaneous clearance. Gut 2004, 53:1673-1681

13. Lavillette D, Morice $Y$, Germanidis G, Donot P, Soulier A, Pagkalos E, Sakellariou G, Intrator L, Bartosch B, Pawlotsky JM, Cosset FL: Human serum facilitates hepatitis $C$ virus infection, and neutralizing responses inversely correlate with viral replication kinetics at the acute phase of hepatitis $C$ virus infection. J Virol 2005, 79(10):6023-6034.

14. Petrovic D, Dempsey E, Doherty DG, Kelleher D, Long A: Hepatitis C virus-T-cell responses and viral escape mutations. Eur J Immuno/ 2012, $42: 17-26$

15. Shoukry NH, Grakoui A, Houghton M, Chien DY, Ghrayeb J, Reimann KA, Walker CM: Memory CD8+ T cells are required for protection from persistent hepatitis C virus infection. J Exp Med 2003, 197:1645-1655.

16. Klenerman $P$, Thimme $R$ : $T$ cell responses in hepatitis $C$ : the good, the bad and the unconventional. Gut 2012, 61:1226-1234.

17. Brown RJ, Tarr AW, McClure CP, Juttla VS, Tagiuri N, Irving WL, Ball JK: Cross-genotype characterization of genetic diversity and molecular adaptation in hepatitis $C$ virus envelope glycoprotein genes. J Gen Virol 2007, 88:458-469.

18. Patronov A, Doytchinova I: T-cell epitope vaccine design by immunoinformatics. Open Bio/ 2013, 3:120139.

19. Dimitrov I, Flower DR, Doytchinova I: Improving in Silico Prediction of Epitope Vaccine Candidates by Union and Intersection of Single Predictors. W J V 2011, 1:15-22.

20. Gededzha MP, Selabe SG, Kyaw T, Rakgole NJ, Blackard JT, Mphahlele MJ: Introduction of new subtypes and variants of hepatitis $C$ virus genotype 4 in South Africa. J Med Virol 2012, 84:601-607.

21. Antaki N, Craxi A, Kamal S, Moucari R, Van der Merwe S, Haffar S, Gadano A, Zein N, Lai CL, Pawlotsky JM, Heathcote EJ, Dusheiko G, Marcellin P: The neglected hepatitis $C$ virus genotypes 4, 5 and 6: an international consensus report. Liver Int 2010, 30:342-355.

22. Bukh J, Apgar CL, Engle R, Govindarajan S, Hegerich PA, Tellier R, Wong DC, Elkins $R$, Kew MC: Experimental infection of chimpanzees with hepatitis $C$ virus of genotype 5a: genetic analysis of the virus and generation of a standardized challenge pool. J Infect Dis 1998, 178:1193-1197.

23. Chamberlain RW, Adams NJ, Taylor LA, Simmonds P, Elliott RM: The complete coding sequence of hepatitis $C$ virus genotype $5 \mathrm{a}$, the predominant genotype in South Africa. Biochem Biophys Res Commun 1997, 236:44-49.

24. Gededzha MP, Selabe SG, Blackard JT, Kyaw T, Mphahlele MJ: Near full-length genome analysis of HCV genotype 5 strains from South Africa. Infect Genet Evol 2014, 21:118-123.

25. Himoudi N, Abraham J, Fournillier A, Lone YC, Joubert A, Op De Beeck A Freida D, Lemonnier F, Kieny MP, Inchauspé G: Comparative vaccine studies in HLA-A2.1-transgenic mice reveal a clustered organization of epitopes presented in hepatitis C virus natural infection. J Virol 2002, 76:12735-12746.

26. Ohno S, Moriya O, Yoshimoto T, Hayashi H, Akatsuka T, Matsui M: Immunogenic variation between multiple HLA-A*0201-restricted, Hepatitis C Virus-derived epitopes for cytotoxic T lymphocytes. Viral Immunol 2006, 19:458-467.

27. Engler OB, Schwendener RA, Dai WJ, Wölk B, Pichler W, Moradpour D, Brunner T, Cerny A: A liposomal peptide vaccine inducing CD8+ T cells in HLA-A2.1 transgenic mice, which recognise human cells encoding hepatitis C virus (HCV) proteins. Vaccine 2004, 23:58-68.

28. Ibe M, Sakaguchi T, Tanaka K, Saito S, Yokota S, Tanaka T, Shimotohno K, Chujoh Y, Shiratori Y, Omata M, Miwa K, Takiguchi M: Identification and characterization of a cytotoxic $T$ cell epitope of hepatitis $C$ virus presented by HLA-B*3501 in acute hepatitis. J Gen Virol 1998, 79:1735-1744.

29. Giugliano S, Oezkan F, Bedrejowski M, Kudla M, Reiser M, Viazov S, Scherbaum N, Roggendorf M, Timm J: Degree of cross-genotype reactivity of hepatitis C virus-specific CD8+ T cells directed against NS3. Hepatology 2009, 50:707-716.

30. Park S, Shin E, Capone S, Caggiari L, Re VD, Nicosia A, Folgori A, Rehermann B: Successful vaccination induces multifunctional memory T-cell precursors associated with early control of hepatitis C virus. Gastroenterology 2012, 143:1048-1060. 
31. Boucherma R, Kridane-Miledi H, Bouziat R, Rasmussen M, Gatard T, Langa-Vives F, Lemercier B, Lim A, Bérard M, Benmohamed L, Buus S, Rooke R, Lemonnier FA: HLA-A*01:03, HLA-A*24:02, HLA-B*08:01, HLA-B*27:05, HLA-B*35:01, HLA-B*44:02, and HLA-C*07:01 monochain transgenic/H-2 class I null mice: novel versatile preclinical models of human $\mathrm{T}$ cell responses. J Immunol 2013, 191:583-593.

32. Doi H, Hiroishi K, Shimazaki T, Eguchi J, Baba T, Ito T, Matsumura T, Nozawa H, Morikawa K, Ishii S, Hiraide A, Sakaki M, Imawari M: Magnitude of CD8 T-cell responses against hepatitis $C$ virus and severity of hepatitis do not necessarily determine outcomes in acute hepatitis $C$ virus infection. Hepatol Res 2009, 39:256-265.

33. Wentworth PA, Sette A, Celis E, Sidney J, Southwood S, Crimi C, Stitely S, Keogh E, Wong NC, Livingston B, Alazard D, Vitiello A, Grey HM, Chisari FV, Chesnut RW, Fikes J: Identification of A2-restricted hepatitis C virus-specific cytotoxic T lymphocyte epitopes from conserved regions of the viral genome. Int Immunol 1996, 8:651-659.

34. Wang S, Buchli R, Schiller J, Gao J, VanGundy RS, Hildebrand WH, Eckels DD: Natural epitope variants of the hepatitis $C$ virus impair cytotoxic $T$ lymphocyte activity. World J Gastroenterol 2010, 16:1953-1969.

35. Fitzmaurice K, Petrovic D, Ramamurthy N, Simmons R, Merani S, Gaudieri S, Sims S, Dempsey E, Freitas E, Lea S, McKiernan S, Norris S, Long A, Kelleher D, Klenerman P: Molecular footprints reveal the impact of the protective HLA-A*03 allele in hepatitis C virus infection. Gut 2011, 60:1563-1571.

36. Scognamiglio P, Accapezzato D, Casciaro MA, Cacciani A, Artini M, Bruno G, Chircu ML, Sidney J, Southwood S, Abrignani S, Sette A, Barnaba V: Presence of effector CD8+ T cells in hepatitis C virus-exposed healthy seronegative donors. J Immunol 1999, 162:6681-6689.

37. Chang KM, Gruener NH, Southwood S, Sidney J, Pape GR, Chisari FV, Sette A: Identification of HLA-A3 and -B7-restricted CTL response to hepatitis C virus in patients with acute and chronic hepatitis C. J Immunol 1999, 162:1156-1164.

38. Sreenarasimhaiah J, Jaramillo A, Crippin J, Lisker-Melman M, Chapman WC, Mohanakumar T: Concomitant augmentation of type 1 CD4+ and CD8+ T-cell responses during successful interferon-alpha and ribavirin treatment for chronic hepatitis C virus infection. Hum Immunol 2003, 64:497-504

39. Matsui M, Moriya O, Belladonna ML, Kamiya S, Lemonnier FA, Yoshimoto T, Akatsuka T: Adjuvant activities of novel cytokines, interleukin-23 (IL-23) and IL-27, for induction of hepatitis C virus-specific cytotoxic T lymphocytes in HLA-A*0201 transgenic mice. J Virol 2004, 78:9093-9104.

40. Mizukoshi E, Nascimbeni M, Blaustein JB, Mihalik K, Rice CM, Liang TJ, Feinstone SM, Rehermann B: Molecular and immunological significance of chimpanzee major histocompatibility complex haplotypes for hepatitis $C$ virus immune response and vaccination studies. J Virol 2002, 76:6093-6103.

41. Zhang J, Yamada O, Yoshida H, Iwai T, Araki H: Autogenous translational inhibition of core protein: implication for switch from translation to RNA replication in hepatitis C virus. Virology 2002, 293:141-150.

42. Matsueda S, Yamada A, Takao Y, Tamura M, Komatsu N, Yutani S, Ide T, Sata M, Itoh $\mathrm{K}$ : A new epitope peptide derived from hepatitis $C$ virus $1 \mathrm{~b}$ possessing the capacity to induce cytotoxic T-lymphocytes in HCV1b-infected patients with HLA-A11, -A31, and -A33. Cancer Immunol Immunother 2007, 56:1359-1366

43. De Groot AS, Sbai H, Aubin CS, McMurry J, Martin W: Immuno-informatics: Mining genomes for vaccine components. Immunol Cell Biol 2002, 80:255-269.

44. Singh H, Raghava GP: ProPred1: prediction of promiscuous MHC Class-I binding sites. Bioinformatics 2003, 19:1009-1014.

45. Singh H, Raghava GP: ProPred: prediction of HLA-DR binding sites. Bioinformatics 2001, 17:1236-1237.

46. Toussaint NC, Dönnes $P$, Kohlbacher O: A mathematical framework for the selection of an optimal set of peptides for epitope-based vaccines. PLoS Comput Biol 2008, 4:e1000246.

47. Zeng R, Li G, Ling S, Zhang H, Yao Z, Xiu B, He F, Huang R, Wei L: A novel combined vaccine candidate containing epitopes of HCV NS3, core and $\mathrm{E} 1$ proteins induces multi-specific immune responses in BALB/c mice. Antiviral Res 2009, 84:23-30.

48. Hu CT: Vaccine Development for Hepatitis C: Lessons from the Past Turn into Promise for the Future. Tzu Chi Med J 2005, 17:61-74

49. Shehzadi A, Ur Rehman S, Idrees M: Promiscuous prediction and conservancy analysis of CTL binding epitopes of HCV 3a viral proteome from Punjab Pakistan: an in silico approach. Virol J 2011, 8:55.
50. Vallet S, Gouriou S, Nousbaum JB, Legrand-Quillien MC, Goudeau A, Picard B: Genetic heterogeneity of the NS3 protease gene in hepatitis C virus genotype 1 from untreated infected patients. J Med Virol 2005, 75(4):528-537.

51. Cento V, Mirabelli C, Salpini R, Dimonte S, Artese A, Costa G, Mercurio F, Svicher V, Parrotta L, Bertoli A, Ciotti M, Di Paolo D, Sarrecchia C, Andreoni M, Alcaro S, Angelico M, Perno CF, Ceccherini-Silberstein F: HCV genotypes are differently prone to the development of resistance to linear and macrocyclic protease inhibitors. PLoS One 2012, 7(7):e39652.

52. Torresi J, Johnson D, Wedemeyer H: Progress in the development of preventive and therapeutic vaccines for hepatitis C virus. J Hepatol 2011, 54:1273-1285.

53. Eckels DD, Bian T, Gill JC, Sønderstrup G: Epitopes of the NS3 protein of hepatitis C virus: recognition in HLA-DR4 transgenic mice. Immunol Cell Biol 2002, 80:106-112.

54. Day CL, Lauer GM, Robbins GK, McGovern B, Wurcel AG, Gandhi RT, Chung RT, Walker BD: Broad specificity of virus-specific CD4+ T-helper-cell responses in resolved hepatitis C virus infection. J Virol 2002, 76:12584-12595.

55. Hakamada T, Funatsuki K, Morita H, Ugajin T, Nakamura I, Ishiko H, Matsuzaki Y, Tanaka N, Imawari M: Identification of novel hepatitis C virus-specific cytotoxic T lymphocyte epitopes by ELISpot assay using peptides with human leukocyte antigen-A*2402-binding motifs. J Gen Virol 2004, 85:1521-1531.

56. Wertheimer AM, Miner C, Lewinsohn DM, Sasaki AW, Kaufman E, Rosen HR: Novel CD4+ and CD8+ T-cell determinants within the NS3 protein in subjects with spontaneously resolved HCV infection. Hepatology 2003, 37:577-589

57. Vertuani S, Bazzaro M, Gualandi G, Micheletti F, Marastoni M, Fortini C, Canella A, Marino M, Tomatis R, Traniello S, Gavioli R: Effect of interferonalpha therapy on epitope-specific cytotoxic T lymphocyte responses in hepatitis C virus-infected individuals. Eur J Immunol 2002, 32:144-154.

58. Smyk-Pearson S, Tester IA, Lezotte D, Sasaki AW, Lewinsohn DM, Rosen HR: Differential antigenic hierarchy associated with spontaneous recovery from hepatitis $C$ virus infection: implications for vaccine design. $J$ Infect Dis 2006, 194:454-463.

59. Hammer J, Belunis C, Bolin D, Papadopoulos J, Walsky R, Higelin J, Danho W, Sinigaglia F, Nagy ZA: High-affinity binding of short peptides to major histocompatibility complex class II molecules by anchor combinations. Proc Natl Acad Sci U S A 1994, 91:4456-4460.

60. Yusim K, Kesmir C, Gaschen B, Addo MM, Altfeld M, Brunak S, Chigaev A, Detours V, Korber BT: Clustering patterns of cytotoxic T-lymphocyte epitopes in human immunodeficiency virus type 1 (HIV-1) proteins reveal imprints of immune evasion on HIV-1 global variation. J Virol 2002, 76:8757-8768.

61. Vider-Shalit T, Raffaeli S, Louzoun Y: Virus-epitope vaccine design: informatic matching the HLA-I polymorphism to the virus genome. Mol Immunol 2007, 44:1253-1261.

62. Molero-Abraham M, Lafuente EM, Flower DR, Reche PA: Selection of conserved epitopes from hepatitis C virus for pan-populational stimulation of T-cell responses. Clin Dev Immunol 2013, 2013:601943.

63. Doytchinova IA, Guan P, Flower DR: EpiJen: a server for multistep T cell epitope prediction. BMC Bioinformatics 2006, 7:131.

64. Hertz T, Nolan D, James I, John M, Gaudieri S, Phillips E, Huang JC, Riadi G, Mallal S, Jojic N: Mapping the landscape of host-pathogen coevolution: HLA class I binding and its relationship with evolutionary conservation in human and viral proteins. J Virol 2011, 85(3):1310-1321.

65. Hall TA: BioEdit: a user-friendly biological sequence alignment editor and analysis program for Windows 95/98/NT. Nucl Acids Symp Ser 1999, 41:95-98.

66. Nielsen M, Lundegaard C, Worning P, Lauemoller SL, Lamberth K, Buus S, Brunak S, Lund O: Reliable prediction of T-cell epitopes using neural networks with novel sequence representations. Protein Sci 2003, 12:1007-1017.

67. Paximadis M, Mathebula TY, Gentle NL, Vardas E, Colvin M, Gray CM, Tiemessen CT, Puren A: Human leukocyte antigen class I (A, B, C) and II (DRB1) diversity in the black and caucasian South African population. Hum Immunol 2012, 73:80-92.

doi:10.1186/1743-422X-11-187

Cite this article as: Gededzha et al:: Prediction of T-cell epitopes of hepatitis C virus genotype 5a. Virology Journal 2014 11:187. 